\title{
Numerical Solutions of Fuzzy Fractional Delay Differential Equations
}

\author{
V. Padmapriya ${ }^{1,2}$, M. Kaliyappan ${ }^{3}$, and A. Manivannan ${ }^{3}$ \\ ${ }^{1}$ Research Scholar, Vellore Institute of Technology, Chennai Campus, India \\ ${ }^{2}$ New Prince Shri Bhavani Arts and Sciences College, Chennai, India \\ ${ }^{3}$ Division of Mathematics, School of Advanced Sciences, Vellore Institute of Technology, Chennai Campus, \\ India
}

\section{]jfis}

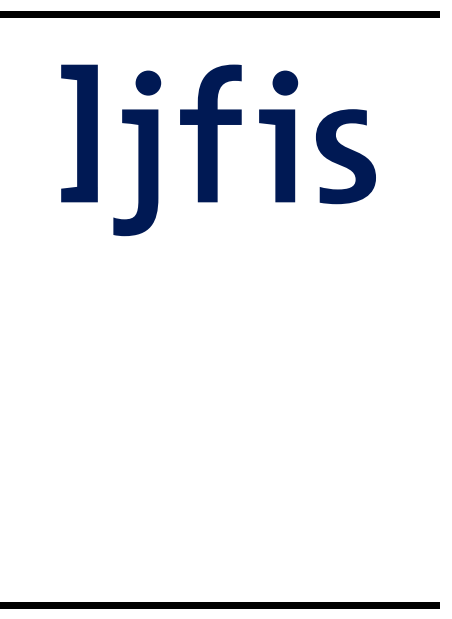

Received: Feb. 28, 2020

Revised : Jun. 10, 2020

Accepted: Aug. 27, 2020

Correspondence to: V. Padmapriya

(v.padmapriya2015@vit.ac.in)

(CThe Korean Institute of Intelligent Systems

cCThis is an Open Access article distributed under the terms of the Creative Commons Attribution Non-Commercial License (http://creativecommons.org/licenses/ by-nc/3.0// which permits unrestricted noncommercial use, distribution, and reproduction in any medium, provided the original work is properly cited.

\begin{abstract}
In this paper, a novel technique is proposed to solve fuzzy fractional delay differential equations (FFDDEs) with initial condition and source function, which are fuzzy triangular functions. The obtained solution is a fuzzy set of real functions. Each real function satisfies an FFDDE with a specific membership degree. A detailed algorithm is provided to solve the FFDDE. The proposed method has been elucidated in detail through numerical illustrations. Graphs are plotted using MATLAB.
\end{abstract}

Keywords: Fractional differential equation (FDE), Fuzzy delay differential equation (FDDE), Fuzzy fractional differential equation (FFDE), Fuzzy fractional delay differential equation (FFDDE), Fuzzy set

\section{Introduction}

Delay differential equation (DDE) is a type of differential equation in which the derivative of an unknown function at any time depends on the solution at prior times. DDE is mainly used in a large number of system models in physics, biology, and engineering. In addition, DDE is applied in many practical systems such as automatic control, traffic models, neuroscience, and lasers. References [1] and [2] presented a complete study of DDEs and their applications.

On the other hand, when a dynamical phenomenon with a delay is modeled using ordinary DDEs, the model is not always accurate. In general, the initial conditions or parameters of the equations are incomplete or vague. This limitation leads to the study of fuzzy DDEs (FDDEs). In recent years, the theory and application of FDDEs have been developed by many researchers. Lupulescu introduced a DDE with a fuzzy case in [3]. Consequently, Khastan et al. [4] and Hoa et al. [5] presented the results of the existence and uniqueness of solutions of generalized FDDEs. Moreover, Hoa et al. [6] introduced fuzzy delay integro-differential equations with generalized Hukuhara differentiability. Random fuzzy delay integro-differential equations were investigated in [7] and [8].

In many real-world tasks, the memory and hereditary properties of differential operators are broadly perceived to be well anticipated through fractional operators. Fractional order is an extension of the classical order of differentiation and integration. In the last few decades, numerous phenomena in different fields of mathematics, economics, engineering, and science have been more precisely described via fractional derivatives, and fractional differential equations (FDEs) have come up as another powerful device for modeling many difficult types 
of complex systems. Podlubny [9] provided a major contribution to this field. The existence, uniqueness result, and some integral transforms of FDEs are presented in [9]. Later, Kilbas et al. [10] presented the idea of fractional calculus. A large amount of literature has been dedicated to the theory and applications of FDEs [11-13]. Currently, fractional chaotic systems are gaining considerable interest among many researchers in the different fields of science, mathematics, and engineering. Huang et al. [14] analyzed the behavior of a chaotic system of a fractional order love model with an external environment. Moreover, Huang et al. [15] studied the relationship of Romeo and Juliet love fractional model using a fuzzy function.

The idea of Riemann-Liouville FDE with an uncertainty was introduced by Agarwal et al. [16]. Consequently, many authors presented results on the existence and uniqueness of solutions for fuzzy FDEs (FFDEs) using numerous methods [1720]. Mazandarani et al. [21] used the modified Euler method to solve FFDEs with a Caputo derivative. Salahshour et al. [22] obtained the solution of FFDEs using the fuzzy Laplace transform method. Ahmed et al. [23] provided analytical and numerical solutions of FFDEs using the Zadeh's extension principle.

In the present work, our aim is to obtain a solution for FDDEs under a fractional derivative. Many authors solved fuzzy fractional DDEs (FFDDEs) using some numerical methods. For instance, Hoa [24] proposed a solution of FFDDEs that involve the Caputo gH-differentiability using the Adams-BashforthMoulton method and also proposed the modified Euler method to solve FFDDEs with an initial condition in [25].

In all the above-cited studies, the source functions and initial conditions are considered as a fuzzy number-valued function. For this function, the derivative is considered as a gH-derivative. This gH-derivative has two types, namely, [(i)-gH] and [(ii)-gH] differentiability. The disadvantages of this derivative are the difficulty in determining which type of derivative to choose and whether the solution is unique. Another difficulty of the gH-derivative is that it may convert the fuzzy problem into two crisp corresponding problems, and we need to choose one solution from a set of solutions that more appropriately fits the problem.

Therefore, our main motivation is to avoid the difficulties in choosing the fuzzy derivative. Then, we enhance the methodology proposed by Fatullayev et al. [26] for FDDEs to FFDDEs. The authors in [26] solved fuzzy problems with an initial condition and a forcing function as a set of fuzzy real functions using the method of Gasilov et al. [27]. Consequently, in [28] the authors provided solutions for fuzzy problems with boundary conditions in which the forcing functions were fuzzy functions.

To the best of our knowledge, this is the first study where this technique is utilized for solving FFDDEs. In the present work, our main objective is to obtain a solution for FDDEs with a fractional derivative. Recently, fuzzy triangular membership function is a beneficial device in many designing applications [29]. Therefore, in this approach, we consider that the initial condition and source functions are in fuzzy triangular forms. Moreover, we prove that if the initial conditions and source functions are fuzzy triangular functions, then the value of the solution is also a fuzzy triangular function. According to this methodology, we present the existence and uniqueness results for of FFDDEs.

This paper is structured as follows: The basic definition and notation of the fractional derivatives, fuzzy sets and fuzzy functions are presented in Section 2. The proposed method for solving FFDDEs is described in Section 3. Numerical applications are performed to test the validity and reliability of the present algorithm in Section 4. Finally, the conclusion is drawn in Section 5.

\section{Basics of Fuzzy and Fractional Calculus}

In this section, we provide certain essential definitions and properties of the fuzzy fractional concept.

Definition $1[9,10]$. The Riemann-Liouville fractional integral of $y(\chi)$ with any order $\alpha>0$ is expressed as

$$
I_{\chi}^{\alpha} y(\chi)=\frac{1}{\Gamma(\alpha)} \int_{0}^{\chi}(\chi-s)^{\alpha-1} y(s) d s .
$$

Definition $2[9,10]$. The Caputo derivative of order $\alpha(0<$ $\alpha<1$ ) of real-valued function $y(\chi)$ is defined as

$$
\begin{aligned}
D^{\alpha} y(\chi) & =I_{\chi}^{1-\alpha}\left[\frac{d y(\chi)}{d \chi}\right] \\
& =\frac{1}{\Gamma(1-\alpha)} \int_{0}^{\chi}(\chi-s)^{-\alpha} \frac{d y(s)}{d s} d s .
\end{aligned}
$$

\subsection{Fuzzy-Set Theory}

We provide fixed set $U$, and a fuzzy set $\tilde{A}$ on $U$ with membership function $\mu_{\tilde{A}}$ can be defined as $\tilde{A}=\left\{\left(x, \mu_{\tilde{A}}(x)\right) \mid x \in U\right\}$.

We also consider $a, b$, and $c$ as real numbers with $a \leq c \leq b$. The membership function of triangular fuzzy number $\tilde{u}=(a$, 
$c, b)$ is defined as

$$
\mu_{\tilde{A}}(x)= \begin{cases}\frac{x-a}{c-a}, & a<x<c, \\ 1, & x=c, \\ \frac{b-x}{b-c}, & c<x<b, \\ 0, & \text { otherwise. }\end{cases}
$$

According to the geometric explanation of a fuzzy number, $c$ is the vertex, $\underline{u}=a$ is the left end point, and $\bar{u}=b$ is the right end point.

In this study, $\tilde{u}$ can be represented by $\tilde{u}=u_{c r}+\tilde{u}_{u n}$, where $u_{c r}$ is the real part $\left(u_{c r}=c\right)$ and $\tilde{u}_{u n}$ is the uncertain part $\left(\tilde{u}_{u n}=(a-c, 0, b-c)\right)$.

A fuzzy set can also be represented through the definition of $r$-cut. For each $0<r \leq 1$, the $r$-cut of $\tilde{A}$ is expressed as $A_{r}=$ $\left\{x \in U \mid \mu_{\tilde{A}}(x) \geq r\right\}$. For $r=0, A_{0}=\operatorname{closure}(\operatorname{support}(\tilde{A}))$, where $\operatorname{support}(\tilde{A})=\left\{x \in U \mid \mu_{\tilde{A}}(x)>0\right\}$.

The $r$-cuts of fuzzy number $\tilde{u}=(a, c, b)$ are intervals $u_{r}=$ $\left[\underline{u}_{r}, \bar{u}_{r}\right]$, where $\underline{u}_{r}=a+r(c-a)$ and $\bar{u}_{r}=b+r(c-b)$. This $\underline{u}_{r}$ and $\bar{u}_{r}$ can be written as $\underline{u}_{r}=c+(1-r)(a-c)$ and $\bar{u}_{r}=c+(1-r)(b-c)$. Then, $\left[\underline{u}_{r}, \bar{u}_{r}\right]=c+(1-r)[a-c$, $b-c]$.

\subsection{Fuzzy Triangular Function}

In our work, we express a fuzzy function as a set of fuzzy real functions in which every real function possesses a specific membership degree. This function is defined by Gasilov et al. [27].

Let $F_{a}(\bullet), F_{b}(\bullet)$, and $F_{c}(\bullet)$ be functions that are continuous on interval $I$.

Fuzzy set $\tilde{F}=\left\langle F_{a}, F_{c}, F_{b}\right\rangle$ is called a fuzzy triangular function if it is determined by the following membership function:

$$
\mu_{\tilde{F}}(y(.))= \begin{cases}r, & y=F_{a}+r\left(F_{c}-F_{a}\right) \text { and } 0<r<1, \\ 1, & y=F_{c}, \\ r, & y=F_{b}+r\left(F_{c}-F_{b}\right) \text { and } 0<r<1, \\ 0, & \text { otherwise. }\end{cases}
$$

The interpretation of triangular fuzzy function $\tilde{F}=\left\langle F_{a}, F_{c}\right.$, $\left.F_{b}\right\rangle$ is presented by Gasilov et al in [27].

The fuzzy triangular function is a fuzzy triangular number for each time $\chi \in I$. Then, we can represent it using the following equation:

$$
\begin{aligned}
\tilde{F}(\chi)= & \left(\min \left\{F_{a}(\chi), F_{c}(\chi), F_{b}(\chi)\right\},\right. \\
& \left.F_{c}(\chi), \max \left\{F_{a}(\chi), F_{c}(\chi), F_{b}(\chi)\right\}\right) .
\end{aligned}
$$

\section{Solution of Fuzzy Fractional Delay Differen- tial Equations}

We consider the following FFDDE:

$$
\begin{cases}{ }^{C} D^{\alpha} y(\chi)=m(\chi) y(\chi-\tau)+\tilde{F}(\chi), & \chi>0,0<\alpha<1, \\ y(\chi)=\tilde{\Phi}(\chi), & -\tau \leq \chi \leq 0,\end{cases}
$$

where $m(\chi)$ is a continuous crisp function and $\tau>0$ is the delay value. $\tilde{\Phi}(\chi)=\left\langle\Phi_{a}, \Phi_{c}, \Phi_{b}\right\rangle$ and $\tilde{F}(\chi)=\left\langle F_{a}, F_{c}, F_{b}\right\rangle$ are triangular fuzzy functions defined on $[-\tau, 0]$ and $(0, \infty)$, respectively, and ${ }^{C} D^{\alpha}$ denotes the Caputo derivative operator.

Definition 3 [26]. Fuzzy set $\tilde{Y}$ is the solution of FFDDE (6) on $U$, and its membership function is defined by

$$
\begin{aligned}
\mu_{\tilde{Y}}(y(\bullet))= & \min \left\{\mu_{\tilde{\Phi}}(\varphi(\bullet)),\right. \\
& \left.\mu_{\tilde{F}}\left({ }^{C} D^{\alpha} y(\chi)-m(\chi) y(\chi-\tau)\right)\right\},
\end{aligned}
$$

where $\varphi(\chi)=y(\chi)$

We can explain Eq. (7) as follows:

Let us consider that $y(\bullet)$ is a function on $U$. Then, we calculate $\varphi(\chi)=y(\chi)$ for $-\tau \leq \chi \leq 0$ and find $\mu_{\tilde{\varphi}}(\varphi(\bullet))=\mu_{1}$. Consequently, for given function $y(\chi)$, we calculate $f(\chi)=$ ${ }^{C} D^{\alpha} y(\chi)-m(\chi) y(\chi-\tau)$ for $\chi>0$ and find $\mu_{\tilde{F}}(f(\bullet))=$ $\mu_{2}$. Next, we determine membership degree $\mu$ for function $y(\bullet)$, which is calculated as $\mu=\min \left\{\mu_{1}, \mu_{2}\right\}$. Then, we define function set $y(\bullet)$ with membership degree $\mu$ as fuzzy solution $\tilde{Y}$

As stated in Definition 3, solution $\tilde{Y}$ is a collection of fuzzy real functions $y(\chi)$. A function $y(\chi)$ has a positive membership degree if it satisfies the following equation:

$$
\begin{cases}{ }^{C} D^{\alpha} y(\chi)=m(\chi) y(\chi-\tau)+f(\chi), & \chi>0,0<\alpha<1, \\ y(\chi)=\varphi(\chi), & -\tau \leq \chi \leq 0,\end{cases}
$$

for $\varphi \in \operatorname{supp}(\tilde{\Phi})$ and $f \in \operatorname{support}(\tilde{F})$.

Let us express $\tilde{F}$ as $\tilde{F}=f_{c r}+\tilde{f}$, where $f_{c r}=F_{c}, \tilde{f}=\left\langle f_{a}\right.$, $\left.0, f_{b}\right\rangle=\left\langle F_{a}-F_{c}, 0, F_{b}-F_{c}\right\rangle$. Let us also express $\tilde{\Phi}$ as $\tilde{\Phi}(\chi)=$ $\varphi_{c r}+\widetilde{\varphi}$, where $\varphi_{c r}=\Phi_{c}, \widetilde{\varphi}=\left\langle\varphi_{a}, 0, \varphi_{b}\right\rangle=\left\langle\Phi_{a}-\Phi_{c}, 0\right.$, $\left.\Phi_{b}-\Phi_{c}\right\rangle$. 
Because Eq. (6) is linear, we apply the principle of superposition into it.

Then, we split FFDDE (6) into three equations as follows:

(1) Fractional non-homogeneous equation

$$
\begin{cases}{ }^{C} D^{\alpha} y(\chi)=m(\chi) y(\chi-\tau)+f_{c r}(\chi), & \chi>0,0<\alpha<1, \\ y(\chi)=\varphi_{c r}(\chi), & -\tau \leq \chi \leq 0,\end{cases}
$$

(2) Fractional homogeneous equation involving an initial function, which is a triangular fuzzy function

$$
\begin{cases}{ }^{C} D^{\alpha} y(\chi)=m(\chi) y(\chi-\tau), & \chi>0,0<\alpha<1, \\ y(\chi)=\tilde{\varphi}(\chi), & -\tau \leq \chi \leq 0 .\end{cases}
$$

(3) Fractional non-homogeneous equation involving a fuzzy source function and whose initial value is zero

$$
\begin{cases}{ }^{C} D^{\alpha} y(\chi)=m(\chi) y(\chi-\tau)+\tilde{f}(\chi), & \chi>0,0<\alpha<1, \\ y(\chi)=0, & -\tau \leq \chi \leq 0 .\end{cases}
$$

Next, we develop an algorithm to solve these three equations.

\subsection{Fractional Non-Homogeneous Equation}

We consider fractional non-homogeneous equation, i.e., Eq. (9). We use the method of steps to solve Eq. (9). First, we obtain the solution in the interval $[0, \tau]$. For $0 \leq \chi \leq \tau$, we obtain $y(\chi-\tau)=\varphi_{c r}(\chi-\tau)$. Then, Eq. 9 9 can be expressed as

$$
\left\{\begin{array}{l}
{ }^{C} D^{\alpha} y(\chi)=m(\chi) \varphi_{c r}(\chi-\tau)+f_{c r}(\chi), \\
y(0)=\varphi_{c r}(0) .
\end{array} .\right.
$$

By performing fractional integration on both sides of Eq. [12], we obtain unique solution $y(\chi)=\varphi_{c r}(0)+J^{\alpha}[m(\chi)$ $\left.\varphi_{c r}(\chi-\tau)\right]+J^{\alpha}\left[f_{c r}(\chi)\right]$ for $0 \leq \chi \leq \tau$, where $J^{\alpha}$ denotes the fractional integral operators.

\subsection{Fractional Homogeneous Equation Involving Initial Fuzzy Function}

Next, we solve Eq. (10) in which the initial condition is fuzzy function $\tilde{\varphi}=\left\langle\varphi_{a}, 0, \varphi_{b}\right\rangle$.

Let us consider that $y_{a}(\chi)$ is the solution of the following equation:

$$
\begin{cases}{ }^{C} D^{\alpha} y(\chi)=m(\chi) y(\chi-\tau), & \chi>0,0<\alpha<1, \\ y(\chi)=\varphi_{a}(\chi), & -\tau \leq \chi \leq 0 .\end{cases}
$$

Let us also consider that $y_{b}(\chi)$ is the solution of the following equation:

$$
\begin{cases}{ }^{C} D^{\alpha} y(\chi)=m(\chi) y(\chi-\tau), & \chi>0,0<\alpha<1, \\ y(\chi)=\varphi_{b}(\chi), & -\tau \leq \chi \leq 0 .\end{cases}
$$

Theorem 1. Let us consider Eq. [10, where $\tilde{\varphi}=\left\langle\varphi_{a}, 0\right.$, $\left.\varphi_{b}\right\rangle$ is a triangular fuzzy function and $m(\chi), \varphi_{a}(\chi)$, and $\varphi_{b}(\chi)$ are continuous functions. If $y_{a}(\chi)$ and $y_{b}(\chi)$ are solutions of Eqs. (13) and (14), respectively, then Eq. (10) has unique solution $\tilde{Y}_{\varphi}=\left\langle y_{a}, 0, y_{b}\right\rangle$.

Proof. According to Definition 3, functions $y_{a}(\chi)$ and $y_{b}(\chi)$ with non-zero membership degree from bunch $\tilde{Y}_{\varphi}$ are solutions to Eqs. (13) and (14) for $\varphi_{a}$ and $\varphi_{b}$ from bunch $\tilde{\varphi}$, respectively.

However, the definition of triangular fuzzy function implies that bunch $\tilde{\varphi}=\left\langle\varphi_{a}, 0, \varphi_{b}\right\rangle$ consists of functions $k \varphi_{a}$ and $k \varphi_{b}$ ( $k=1-r$ and $0 \leq r \leq 1)$ because $\Phi_{c}=0$.

Moreover, we apply the linear property for the fractional order (see [30]). Then, if $y(\chi)$ is a solution function of ${ }^{C} D^{\alpha} y(\chi)$ $=m(\chi) y(\chi-\tau)$ with initial condition $y(\chi)=\varphi(\chi)$, then $k y(\chi)$ is a solution function of ${ }^{C} D^{\alpha} y(\chi)=m(\chi) y(\chi-\tau)$ with initial condition $y(\chi)=k \varphi(\chi)$, where $k$ is a constant number.

This result implies that set $\tilde{Y}_{\varphi}$ contains functions $k y_{a}$ and $k y_{b}(0 \leq k \leq 1)$. Hence, $\tilde{Y}_{\varphi}$ is a fuzzy triangular function derived by $\tilde{Y}_{\varphi}=\left\langle y_{a}, 0, y_{b}\right\rangle$. Thus, the theorem is proven.

Remark 1. Fuzzy triangular function $\tilde{Y}_{\varphi}=\left\langle y_{a}, 0, y_{b}\right\rangle$ can be expressed as a triangular fuzzy number at time $\chi$ as follows:

$$
\tilde{Y}_{\varphi}(\chi)=\left\{\min \left\{y_{a}(\chi), 0, y_{b}(\chi)\right\}, 0, \max \left\{y_{a}(\chi), 0, y_{b}(\chi)\right\}\right\}
$$

\subsection{Fractional Non-Homogeneous Equation Involving} Fuzzy Source Function and Zero Initial Value

Next, we solve Eq. 111 in which fuzzy source function $\tilde{f}$ $=\left\langle f_{a}, 0, f_{b}\right\rangle$ and the initial condition is zero.

We let $y_{u}(\chi)$ be the solution to the following problem:

$$
\begin{cases}{ }^{C} D^{\alpha} y(\chi)=m(\chi) y(\chi-\tau)+f_{a}(\chi), & \chi>0,0<\alpha<1, \\ y(\chi)=0, & -\tau \leq \chi \leq 0 .\end{cases}
$$


We also let $y_{v}(\chi)$ be the solution to the following problem:

$$
\begin{cases}{ }^{C} D^{\alpha} y(\chi)=m(\chi) y(\chi-\tau)+f_{b}(\chi), & \chi>0,0<\alpha<1, \\ y(\chi)=0, & -\tau \leq \chi \leq 0 .\end{cases}
$$

Theorem 2. Let us consider Eq. (11). Here, $\tilde{f}=\left\langle f_{a}, 0, f_{b}\right\rangle$ is a triangular fuzzy function, and $m(\chi), f_{a}(\chi)$, and $f_{b}(\chi)$ are continuous functions. If $y_{u}(\chi)$ and $y_{v}(\chi)$ are solutions of Eqs. (15) and (16), respectively, then Eq. (11) has unique solution $\tilde{Y}_{f}=\left\langle y_{u}, 0, y_{v}\right\rangle$.

Proof. The proof of this theorem can be derived from the same procedure used in Theorem 1.

\subsection{Algorithm for the Solution}

Next, we provide the algorithm for solving FFDDE (6).

Step 1: We express the source function and initial value as

$$
\tilde{F}(\chi)=f_{c r}+\left\langle f_{a}, 0, f_{b}\right\rangle \text { and } \tilde{\Phi}(\chi)=\varphi_{c r}+\left\langle\varphi_{a}, 0, \varphi_{b}\right\rangle \text {. }
$$

Step 2: We find solution $y_{c r}(\chi)$ of Eq. (9) using the step method or any other numerical methods.

Step 3: We determine solutions $y_{a}(\chi)$ and $y_{b}(\chi)$ to Eqs. (13) and (14), respectively. Then, the solution of Eq. (10) can be defined as

$$
\tilde{Y}_{\varphi}(\chi)=\left\{\min \left\{y_{a}(\chi), 0, y_{b}(\chi)\right\}, 0, \max \left\{y_{a}(\chi), 0, y_{b}(\chi)\right\}\right\}
$$

Step 4: We find solutions $y_{u}(\chi)$ and $y_{v}(\chi)$ to Eqs. 15 and (16), respectively. Then, the solution of Eq. (11) can be expressed as

$\tilde{Y}_{f}(\chi)=\left\{\min \left\{y_{u}(\chi), 0, y_{v}(\chi)\right\}, 0, \max \left\{y_{u}(\chi), 0, y_{v}(\chi)\right\}\right\}$

Step 5: The solution of FFDDE (6) is expressed as follows:

$$
\tilde{Y}(\chi)=y_{c r}(\chi)+\tilde{Y}_{\varphi}(\chi)+\tilde{Y}_{f}(\chi) .
$$

\section{Example}

Let us consider the following FFDDE:

$$
\left\{\begin{array}{c}
{ }^{C} D^{\alpha} y(\chi)=\lambda \chi y(\chi-1)+\left\langle\chi^{2}, 2 \chi^{2}, 3 \chi^{2}\right\rangle, \\
\chi \in[0,1], 0<\alpha<1, \\
y(\chi)=\tilde{\Phi}(\chi)=\langle\chi+1,2 \chi+2,3 \chi+3\rangle, \\
\chi \in[-1,0],
\end{array}\right.
$$

where $\lambda \in[-1,1] \backslash\{0\}$.

We represent the source function and initial value as $\tilde{F}(\chi)=$ $f_{c r}+\left\langle f_{a}, 0, f_{b}\right\rangle=2 \chi^{2}+\left\langle-\chi^{2}, 0, \chi^{2}\right\rangle$ and $\tilde{\Phi}(x)=\varphi_{c r}+\left\langle\varphi_{a}\right.$, $\left.0, \varphi_{b}\right\rangle=2 \chi+2+\langle-\chi-1,0, \chi+1\rangle$.

First, we find a solution to the following fractional associated non-homogeneous equation:

$$
\begin{cases}{ }^{C} D^{\alpha} y(\chi)=\lambda \chi y(\chi-1)+f_{c r}, & \chi \in[0,1], 0<\alpha<1, \\ y(\chi)=\varphi_{c r}=2 \chi+2, & \chi \in[-1,0],\end{cases}
$$

where $f_{c r}=2 \chi^{2}$.

By solving Eq. [19], we obtain the following solution:

$$
y_{c r}(\chi)=2+\frac{4(\lambda+1)}{\Gamma(3+\alpha)} \chi^{2+\alpha} .
$$

Solution $y_{c r}(\chi)$ is shown in Figure 1 (dashed black color line).

Second, we derive the solution to the fractional homogeneous equation involving fuzzy initial function

$$
\left\{\begin{aligned}
{ }^{C} D^{\alpha} y(\chi)=\lambda \chi y(\chi-1), & \chi \in[0,1], 0<\alpha<1, \\
y(\chi) & =\left\langle\varphi_{a}, 0, \varphi_{b}\right\rangle \\
& =\langle-\chi-1,0, \chi+1\rangle, \quad \chi \in[-1,0] .
\end{aligned}\right.
$$

Then, we solve the following fractional equation:

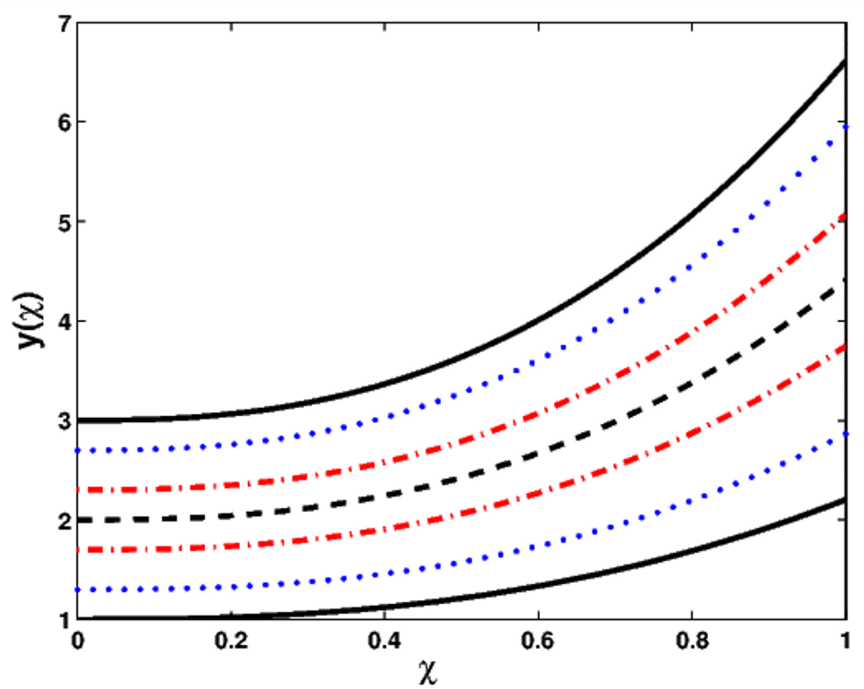

Figure 1. Solution function and $r$-cuts: black color line-0-cut; dotted blue color line - 0.3 -cut; dashed-dotted red color line - 0.7 -cut; and dashed black color line-1.0-cut. $\lambda=1$ and $\alpha=0.5$. 


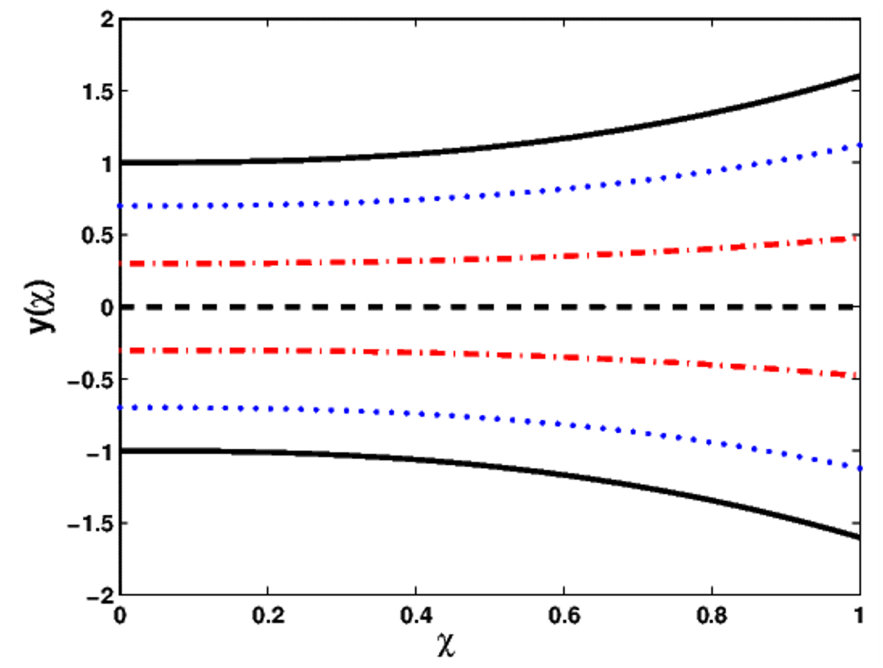

Figure 2. Fuzzy solution in terms of the initial values and $r$-cuts: black color line - 0 -cut; dotted blue color line - 0.3 -cut; dasheddotted red color line -0.7 -cut; and dashed black color line-1.0-cut. $\lambda=1$ and $\alpha=0.5$.

$$
\begin{cases}{ }^{C} D^{\alpha} y(\chi)=\lambda \chi y(\chi-1), & \chi \in[0,1], 0<\alpha<1 \\ y(\chi)=\varphi(\chi), & \chi \in[-1,0]\end{cases}
$$

For $\varphi(\chi)=\varphi_{a}(\chi)$, we obtain solution $y_{a}(\chi)=-1-$ $\frac{2 \lambda}{\Gamma(3+\alpha)} \chi^{2+\alpha}$, and for $\varphi(\chi)=\varphi_{b}(\chi)$, we obtain solution $y_{b}(\chi)$ $=1+\frac{2 \lambda}{\Gamma(3+\alpha)} \chi^{2+\alpha}$.

The obtained solution from Eq. (21) represents triangular fuzzy function $\tilde{Y}_{\varphi}=\left\langle y_{a}, 0, y_{b}\right\rangle$. Solution $\tilde{Y}_{\varphi}$ and its $r$-cuts are shown in Figure 2, and for time $\chi$, we obtain

$$
\tilde{Y}_{\varphi}(\chi)=\left(\min \left\{y_{a}(\chi), 0, y_{b}(\chi)\right\}, 0, \max \left\{y_{a}(\chi), 0, y_{b}(\chi)\right\}\right)
$$

Next, we derive the solution for the fractional non-homogeneous equation involving a fuzzy source function.

$$
\begin{cases}{ }^{C} D^{\alpha} y(\chi)=\lambda \chi y(\chi-1)+\left\langle f_{a}, 0, f_{b}\right\rangle, & \chi \in[0,1], 0<\alpha<1, \\ y(\chi)=0, & \chi \in[-1,0] .\end{cases}
$$

Then, we solve the crisp problems.

$$
\begin{cases}{ }^{C} D^{\alpha} y(\chi)=\lambda \chi y(\chi-1)+f(\chi), & \chi \in[0,1], 0<\alpha<1, \\ y(\chi)=0, & \chi \in[-1,0] .\end{cases}
$$

For $f(\chi)=f_{a}(\chi)$, we obtain solution $y_{u}(\chi)=\frac{-2}{\Gamma(3+\alpha)} \chi^{2+\alpha}$, and for $f(\chi)=f_{b}(\chi)$, we obtain solution $y_{v}(\chi)=\frac{2}{\Gamma(3+\alpha)} \chi^{2+\alpha}$.

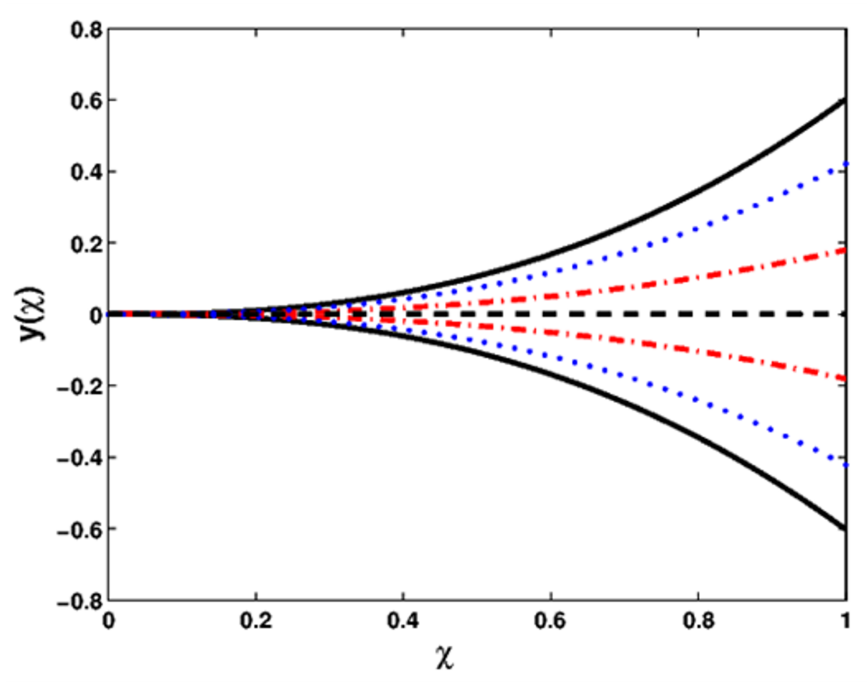

Figure 3. Fuzzy solution relative to the source function and its $r$-cuts: black color line - 0 -cut; dotted blue color line - 0.3 - cut; dasheddotted red color line- -0.7 -cut; and dashed black color line-1.0-cut $\lambda=1$ and $\alpha=0.5$.

The obtained solution from Eq. 24 is fuzzy triangular function $\tilde{Y}_{f}=\left\langle y_{u}, 0, y_{v}\right\rangle$. Solution $\tilde{Y}_{f}$ and its $r$-cuts are shown in Figure 3. For time $t$, we obtain

$\tilde{Y}_{f}(\chi)=\left(\min \left\{y_{u}(\chi), 0, y_{v}(\chi)\right\}, 0, \max \left\{y_{u}(\chi), 0, y_{v}(\chi)\right\}\right)$.

Finally, we derive the solution of Equation $(18)$ in the following form:

$$
\tilde{Y}(\chi)=y_{c r}(\chi)+\tilde{Y}_{\varphi}(\chi)+\tilde{Y}_{f}(\chi) .
$$

Solution $\tilde{Y}(\chi)$ and its $r$-cuts are shown in Figure 1 .

\section{Conclusion}

In this study, we obtained the solutions of an FFDDE according to the method proposed by Fatullayev et al. [26]. In the present work, we considered that the initial condition and source function are triangular fuzzy functions. Meanwhile, the FFDDE was converted into three equations based on the initial and source functions. Finally, we combined all the solutions of the abovementioned three equations to obtain a solution of the FFDDE. Graphs were plotted for the fuzzy solution. The obtained solutions for the FFDDE were expressed as a fuzzy set of real functions. We recommended an efficient method to compute the fuzzy solution set. The numerical example demonstrated that the proposed method is efficient and accurate for application to 
FFDDEs.

\section{Conflict of Interest:}

No potential conflict of interest relevant to this article was reported.

\section{References}

[1] J. K. Hale, Theory of Functional Differential Equations, Springer, New York, 1977.

[2] Y. Kuang, Delay Differential Equations with Applications in Population Dynamics, Academic Press, Boston, 1993.

[3] V. Lupulescu, "On a class of fuzzy functional differential equations," Fuzzy Sets and Systems, vol. 160, pp. 15471562, 2009.

[4] A. Khastan, J. J. Nieto, and R. Rodríguez-López, “Fuzzy delay differential equations under generalized differentiability," Information Sciences, vol. 275, pp. 145-167, 2014.

[5] N. V. Hoa, P. Van Tri, T. T. Dao, and I. Zelinka, "Some global existence results and stability theorem for fuzzy functional differential equations," Journal of Intelligent \& Fuzzy Systems, vol. 28, no. 1, pp. 393-409, 2015.

[6] N. V. Hoa and N. D. Phu, "Fuzzy functional integro-differential equations under generalized $\mathrm{H}$ differentiability," Journal of Intelligent and Fuzzy Systems, vol. 26, no. 4, pp. 2073-2085, 2014.

[7] H. Vu, L. S. Dong, and N. V.Hoa, "Random fuzzy functional integro-differential equations under generalized Hukuhara differentiability," Journal of Intelligent and Fuzzy Systems, vol. 27, no. 3, pp. 1491-1506, 2014.

[8] H. Vu, N. V. Hoa, and N. D. Phu, "The local existence of solutions for random fuzzy integro-differential equations under generalized H-differentiability," Journal of Intelligent and Fuzzy Systems, vol. 26, no. 6, pp. 2701-2717, 2014.

[9] I. Podlubny, Fractional Differential Equation, San Diego: Academic Press, 1999.

[10] A. A. A. Kilbas, H. M. Srivastava, and J. J. Trujillo, Theory and applications of fractional differential equations, Vol. 204, Elsevier Science Limited, 2006.
[11] T. Gnana Bhaskar, V. Lakshmikantham, and S. Leela, "Fractional differential equations with a KrasnoselskiiKrein type condition," Nonlinear Analysis: Hybrid Systems, vol. 3, pp. 734-737, 2009.

[12] V. Lakshmikantham and S. Leela, "A Krasnoselskii-Kreintype uniqueness result for fractional differential equations," Nonlinear Analysis, vol. 71, pp. 3421-3424, 2009.

[13] V. Lakshmikantham, "Theory of fractional functional differential equations," Nonlinear Analysis, vol. 69, pp. $3337-$ 43, 2008.

[14] L. Huang and Y. Bae, "Chaotic Dynamics of the Fractional-Love Model with an External Environment,' Entropy, vol. 20, no. 1, p. 53, 2018.

[15] L. Huang and Y. Bae, "Nonlinear Behavior in FractionalOrder Romeo and Juliet's Love Model Influenced by External Force with Fuzzy Function," International Journal of Fuzzy Systems, vol. 21, no. 2, pp. 630-638, 2019.

[16] R. P.Agarwal, V. Lakshmikantham, and J. J. Nieto, “On the concept of solution for fractional differential equations with uncertainty," Nonlinear Analysis, vol. 72, pp. 2859 2862, 2010.

[17] S. Arshad and V. Lupulescu, "On the fractional differential equations with uncertainty," Nonlinear Analysis, vol. 74, pp 85-93, 2011.

[18] S. Salahshour, T. Allahviranloo, S. Abbasbandy, and D. Baleanu, "Existence and Uniqueness results for fractional differential equations with uncertainty," Advances in Difference Equations, vol. 2012, pp. 1-12, 2012.

[19] T. Allahviranloo, S. Salahshour, and S. Abbasbandy, "Explicit solutions of fractional differential equations with uncertainty," Soft Computing, vol. 16, no. 2, pp. 297-302, 2012.

[20] R. Alikhani and F. Bahrami, "Global solutions for nonlinear fuzzy fractional integral and integro-differential equations," Communications in Nonlinear Science and Numerical Simulation, vol. 18, no. 8, pp. 2007-2017, 2013.

[21] M. Mazandarani and A. V. Kamyad, "Modified fractional Euler method for Solving fuzzy fractional initial value problem," Communications in Nonlinear Science and $\mathrm{Nu}$ merical Simulation, vol. 18, pp. 12-21, 2013. 
[22] S. Salahshour, T. Allahviranloo, and S. Abbasbandy, "Solving fuzzy fractional differential equations by fuzzy Laplace transforms," Communications in Nonlinear Science and Numerical Simulation, vol. 17, pp. 1372-81, 2012.

[23] M. Z. Ahmad, M. K. Hasan, and S. Abbasbandy, "Solving fuzzy fractional differential equations using Zadeh's extension principle," The Scientific World Journal, vol. 2013, pp. 1-11, 2013.

[24] N. V. Hoa, "Fuzzy fractional functional differential equations under Caputo gH-differentiability," Communications in Nonlinear Science and Numerical Simulation, vol. 22, no. 1-3, pp. 1134-1157, 2015.

[25] V. H. Ngo, "Fuzzy fractional functional integral and differential Equations," Fuzzy Sets and Systems, vol. 280, pp. 58-90, 2015.

[26] A. G. Fatullayev, N. A. Gasilov, and S. E. Amrahov, "Numerical solution of linear inhomogeneous fuzzy delay differential equations," Fuzzy Optimization and Decision Making, pp. 1-12, 2018.

[27] N. A. Gasilov, I. F. Hashimoglu, S. E. Amrahov, and A. G. Fatullayev, "A new approach to nonhomogeneous fuzzy initial value problem," Computer Modeling in Engineering \& Sciences(CMES), vol. 85, no. 4, pp. 367-378, 2012.

[28] N. A. Gasilov, S. E. Amrahov, A. G. Fatullayev, and I. F. Hashimoglu, "Solution method for a boundary value problem with fuzzy forcing function," Information Sciences, vol. 317, pp. 349-368, 2015.

[29] W. Pedrycz, "Why triangular membership functions?," Fuzzy Sets and System, vol. 64, no. 1, pp. 21-30, 1994.

[30] B. Bonilla, M. Rivero, and J. J. Trujillo, "Linear differential equations of fractional order," Advances in Fractional Calculus, Springer Netherlands, pp. 77-91, 2007.

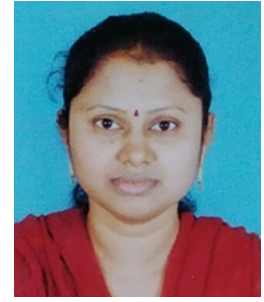

V. Padmapriya is a Ph.D Research scholar in Division of Mathematics, Vellore Institute of Technology-Chennai Campus, India. She is working as Assistant professor in Department of Statistics, New prince Shri Bhavani Arts and Science College, Chennai, India. Her research interest includes Fuzzy differential equations and Fuzzy fractional differential equations.

E-mail: v.padmapriya2015@vit.ac.in

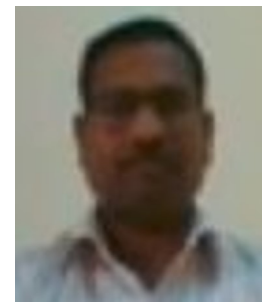

M. Kaliyappan is working as a Professor of Mathematics, Division of mathematics, School of Advanced Sciences, VIT Chennai, India. He has over 23 years of experience in teaching and research. His research interest includes Approximation theory, Numerical computing, Differential equations, Fuzzy differential equations, Fractional differential equations, Fuzzy fractional differential equations and Optimization theory.

E-mail: kaliyappan.m@vit.ac.in

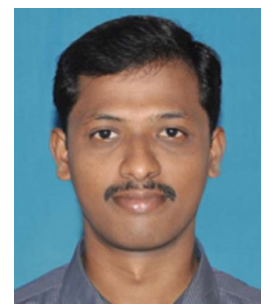

A. Manivannan received the Ph.D. degree in Mathematics from the Madurai Kamaraj University, Madurai, Tamilnadu, India in 2015. He is currently an Assistant Professor in the Division of Mathematics, Vellore Institute of Technology-Chennai Campus, India. His research interests include Stability theory, Neural networks, Complex systems, Fuzzy functional differential equations, Fractional differential equations.

E-mail: manivannan.a@vit.ac.in 